\title{
Superconducting properties of mesoscopic cylinders with enhanced surface superconductivity
}

\author{
B. J. Baelus, S. V. Yampolskii, ${ }^{*}$ and F. M. Peeters ${ }^{\dagger}$ \\ Departement Natuurkunde, Universiteit Antwerpen (UIA), Universiteitsplein 1, B-2610 Antwerpen, Belgium \\ E. Montevecchi and J. O. Indekeu \\ Laboratorium voor Vaste-Stoffysica en Magnetisme, Katholieke Universiteit Leuven, B-3001 Leuven, Belgium
}

(Received 20 June 2001; published 19 December 2001)

\begin{abstract}
The superconducting state of an infinitely long superconducting cylinder surrounded by a medium which enhances its superconductivity near the boundary is studied within the nonlinear Ginzburg-Landau theory. This enhancement can be due to the proximity of another superconductor or due to surface treatment. Quantities such as the free energy, the magnetization and the Cooper-pair density are calculated. Phase diagrams are obtained to investigate how the critical field and the critical temperature depend on this surface enhancement for different values of the Ginzburg-Landau parameter $\kappa$. Increasing the superconductivity near the surface leads to higher critical fields and critical temperatures. For small cylinder diameters only giant vortex states nucleate, while for larger cylinders multivortices can nucleate. The stability of these multivortex states also depends on the surface enhancement. For type-I superconductors we found the remarkable result that for a range of values of the surface extrapolation length the superconductor can transit from the Meissner state into superconducting states with vorticity $L>1$. Such a behavior is not found for the case of large $\kappa$, i.e., type-II superconductivity.
\end{abstract}

DOI: 10.1103/PhysRevB.65.024510

PACS number(s): 74.60.Ec, 74.20.De, 74.25.Dw

\section{INTRODUCTION}

The study of the superconducting properties of mesoscopic samples received recently a lot of attention due to the progress in nanofabrication technologies. A sample is mesoscopic if its size is comparable to the penetration depth $\lambda$ and/or the coherence length $\xi$.

The properties of superconductors and therefore their applications are determined by the critical parameters, i.e. the critical fields $\left(H_{c}, H_{c 1}, H_{c 2}, H_{c 3}\right)$, the critical current $j_{c}$, and the critical temperature $T_{c}$. For application purposes one wants to improve these parameters. Instead of searching for new bulk materials with new physical properties, one can also try to modify the properties of an existing material by nanostructuring the superconductor, i.e., "quantum design." Therefore, theoretical and experimental studies directed to improve the critical parameters of mesoscopic superconductors are important.

From the point of view of improving these parameters, mesoscopic thin disks immersed in an insulating medium and placed in a perpendicular magnetic field have attracted most attention. In disks with a small radius only giant vortex states exist which are axially symmetric superconducting states. ${ }^{1-4}$ The different giant vortex states are characterized by their angular momentum, i.e., their vorticity $L$. For disks with a sufficiently large radius, multivortex states can exist which are the equivalent of the Abrikosov vortex lattice in bulk type-II superconductors $(\kappa=\lambda / \xi>1 / \sqrt{2})$ and the vorticity corresponds to the number of separate vortices. ${ }^{5-18}$ For such disks, it is found that the critical magnetic field depends strongly on the size and on the Ginzburg-Landau parameter and less on the thickness of the disk.

Fink and Presson ${ }^{19,20}$ studied the Meissner state and the giant vortex state of a superconducting infinite cylinder immersed in an insulator or vacuum and placed in a magnetic field parallel to the cylinder axis using the Ginzburg-Landau theory. They calculated how the critical field depends on the cylinder radius and on the Ginzburg-Landau parameter $\kappa$. Zharkov et al. ${ }^{21}$ studied similar superconducting cylinders using the Ginzburg-Landau theory and calculated the critical fields as a function of the cylinder radius, the temperature, and the Ginzburg-Landau parameter.

Varying the sample size and the Ginzburg-Landau parameter, one can decrease or increase the critical field, and thus the critical current, but it does not influence the critical temperature. How can one increase the critical temperature? Fink and Joiner ${ }^{22}$ considered a semi-infinite superconducting half-space where the surface was treated by cold working in such a way that the superconductivity near the surface was enhanced, i.e., the slope of the superconducting order parameter increased near the sample surface. They found that such a surface treatment leads to higher critical temperatures, larger critical fields, and larger critical currents. Another way to enhance superconductivity near the surface is by bringing the superconductor in contact with a well chosen superconducting or semiconducting layer. ${ }^{23}$ The added superconducting layer must have a higher transition temperature than the superconducting sample and in this case the surface enhancement of superconductivity is caused by the proximity effect. It is necessary that this semiconducting layer has a bandgap which overlaps the superconducting gap of the superconductor.

Montevecchi and Indekeu ${ }^{24}$ performed a theoretical study of the effect of confinement on the superconducting/normal transition for systems with surface enhancement. Using the linear Ginzburg-Landau theory they studied the critical temperature at zero field for a thin film, an infinite cylinder, and a sphere. For all geometries they found that at $H=0$ the critical temperature increases with the enhancement of surface superconductivity. For thin films in a parallel field, they 
also calculated a $H-T$ phase diagram. They found that surface enhancement leads to an increase of the critical field $H_{c 3}$ and of the critical temperature.

Yampolskii and Peeters ${ }^{25}$ investigated theoretically the vortex structure of thin mesoscopic disks in a perpendicular magnetic field surrounded by a medium which enhances surface superconductivity. If the size of these thin disks is sufficiently large, they found giant vortex states as well as multivortex states appearing as metastable states and as ground states. The enhancement of the superconductivity near the surface leads to a stabilization of the multivortex states as ground states. They also calculated a $H-T$ phase diagram which showed that the critical temperature and the critical field were significantly increased by enhancing the surface superconductivity.

In the present paper we investigate the effect of the enhancement of surface superconductivity on the critical field and the critical temperature for superconducting cylinders with radii equal to a few coherence lengths $\xi$. We also study the influence of the Ginzburg-Landau parameter $\kappa$. Our theoretical analysis is based on a full self-consistent numerical solution of the coupled nonlinear Ginzburg-Landau equations. No a priori arrangement of the vortex configuration or of the type of vortex configuration is assumed.

The paper is organized as follows. In Sec. II we present our theoretical model. We explain how the enhancement of superconductivity near the surface is taken into account. In Sec. III we consider small cylinders, i.e., infinitely long superconducting cylinders with small radius. In such small cylinders only axially symmetric states or giant vortex states nucleate. We discuss how the critical field and the critical temperature are influenced by the surface enhancement of superconductivity. In Sec. IV larger cylinders are studied where multivortices appear. We investigate the dependence of the nucleation of these states on the surface enhancement. Our results are summarized in Sec. V.

\section{THEORETICAL FORMALISM}

We consider infinite superconducting cylinders with radius $R$ surrounded by a medium which enhances superconductivity at the edge of the cylinder. Along the axis of the cylinder a uniform magnetic field $\vec{H}_{0}$ is applied. To deal with this problem we use the Ginzburg-Landau theory and we solve numerically and self-consistently the system of two coupled equations

$$
\begin{gathered}
\frac{1}{2 m}\left(-i \hbar \vec{\nabla}-\frac{2 e \vec{A}}{c}\right)^{2} \Psi=-\alpha \Psi-\beta \Psi|\Psi|^{2}, \\
\vec{\nabla} \times(\vec{\nabla} \times \vec{A})=\frac{4 \pi}{c} \vec{j},
\end{gathered}
$$

where the density of superconducting current $\vec{j}$ is given by

$$
\vec{j}=\frac{e \hbar}{i m}\left(\Psi * \vec{\nabla} \Psi-\Psi \vec{\nabla} \Psi^{*}\right)-\frac{4 e^{2}}{m c}|\Psi|^{2} \vec{A} .
$$

The boundary condition for the vector potential is such that far away from the cylinder the field equals the external field $H_{0}$, i.e.,

$$
\left.\vec{A}\right|_{\rho \rightarrow \infty}=\frac{1}{2} H_{0} \rho \vec{e}_{\phi},
$$

where $\rho$ indicates the radial position from the center of the cylinder and $\vec{e}_{\phi}$ is the azimuthal direction. The general boundary condition for the order parameter can be written $\mathrm{as}^{26}$

$$
\left.\vec{n}\left(-i \hbar \vec{\nabla}-\frac{2 e \vec{A}}{c}\right) \Psi\right|_{\rho=R}=\left.\frac{i}{b} \Psi\right|_{\rho=R},
$$

where $\vec{n}$ is the unit vector normal to the cylinder and $b$ is the surface extrapolation length. The value of $b$ is determined by the medium in which the cylinder is immersed. If the surrounding medium is vacuum or an insulator one has $b \rightarrow \infty$, for metals $b>0$ and for ferromagnets $b \rightarrow 0$. When, for example, the surface of the cylinder is in contact with a superconducting layer of a higher $T_{c}$ one has $b<0$. In the present paper we will consider the latter case of $b<0$.

Using dimensionless variables and the London gauge div $\vec{A}=0$ the GL equations can be written as

$$
\begin{gathered}
(-i \vec{\nabla}-\vec{A})^{2} \Psi=\Psi\left(1-|\Psi|^{2}\right), \\
-\kappa^{2} \Delta \vec{A}=\frac{1}{2 i}\left(\Psi^{*} \vec{\nabla} \Psi-\Psi \vec{\nabla} \Psi^{*}\right)-|\Psi|^{2} \vec{A},
\end{gathered}
$$

and the boundary condition for the order parameter as

$$
\left.\vec{n}(-i \vec{\nabla}-\vec{A}) \Psi\right|_{\rho=R}=\left.\frac{i}{b} \Psi\right|_{\rho=R} .
$$

Here the distance is measured in units of the coherence length $\xi$, the order parameter in $\Psi_{0}=\sqrt{-\alpha / \beta}$, the vector potential in $c \hbar / 2 e \xi$, and the magnetic field in $H_{c 2}$ $=c \hbar / 2 e \xi^{2}=\kappa \sqrt{2} H_{c}$, where $H_{c}=\sqrt{4 \pi \alpha^{2} / \beta}$ is the critical field.

To solve the system of Eqs. (3a), (3b), we apply a finitedifference representation of the order parameter and the vector potential on a uniform Cartesian space grid $(x, y)$, with typically 128 grid points over a distance $2 R_{0}$ (i.e., the diameter of the cylinder), and we used the link variable approach, ${ }^{27}$ and an iteration procedure based on the GaussSeidel technique to find $\Psi$. The vector potential is obtained with the fast Fourier transform technique where we set $\vec{A}_{|x|=R_{S},|y|=R_{S}}=H_{0}(x,-y) / 2$ at the boundary of a larger space grid (typically $R_{S}=4 R_{0}$ ).

Two different vortex configurations are possible in axial symmetric samples; the giant vortex state and the multivortex state. The giant vortex state has cylindrical symmetry, which leads to the order parameter $\Psi=\psi(\rho) \exp (i L \phi)$, where $\rho$ and $\phi$ are the cylindrical coordinates and $L$ is the angular momentum or vorticity. Along a closed path, which lies near 
the edge of the cylinder, the order parameter phase difference is given by $L$ times $2 \pi$. A multivortex state is described by a mixture of different angular harmonics which is possible due to the nonlinearity of the GL equations. In this case the vorticity is nothing else than the number of vortices in the superconductor.

To find the different vortex configurations, which include the metastable states, we search for the steady-state solutions of Eqs. (3a), (3b) starting from different randomly generated initial conditions. Then we increase/decrease slowly the magnetic field and recalculate each time the exact vortex structure. We do this for each vortex configuration in a magnetic field range where the vorticity stays the same.

In order to determine which vortex state corresponds to the ground state we calculated the difference in free energy density between the superconducting state and the normal state

$$
\begin{aligned}
\frac{F}{F_{0}}= & \frac{2}{V} \int_{V} d V\left[-|\Psi|^{2}+\frac{1}{2}|\Psi|^{4}+|(-i \vec{\nabla}-\vec{A}) \Psi|^{2}+\kappa^{2}(\vec{H}\right. \\
& \left.\left.-\vec{H}_{0}\right)^{2}\right]+\frac{2}{b V} \oint_{S} d S|\Psi|^{2},
\end{aligned}
$$

where $\vec{H}=\operatorname{rot} \vec{A}, V$ is the volume of a cylinder with unit height, $S$ the surface of the infinite cylinder, and $F_{0}$ $=H_{c}^{2} V / 8 \pi$. The contribution of the surface is taken into account by the last term of Eq. (5). By comparing the dimensionless free energy of the different giant and multivortex configurations, we obtain the ground states and the metastable states. The dimensionless magnetization is defined as

$$
M=\frac{\langle H\rangle-H_{0}}{4 \pi},
$$

where $\langle H\rangle$ is the magnetic field averaged over the sample. The magnetization is a direct measure of the magnetic field expelled from the sample.

The temperature is included in $\xi, \lambda, H_{c 2}$, through their temperature dependencies

$$
\begin{gathered}
\xi(T)=\frac{\xi(0)}{\sqrt{\left|1-T / T_{c 0}\right|}}, \\
\lambda(T)=\frac{\lambda(0)}{\sqrt{\left|1-T / T_{c 0}\right|}}, \\
H_{c 2}(T)=H_{c 2}(0) \mid 1-\frac{T}{T_{c 0} \mid},
\end{gathered}
$$

where $T_{c 0}$ is the critical temperature at zero magnetic field for the normal boundary condition, i.e., $-\xi(0) / b=0$. We will only insert the temperature explicitly if we consider the $H-T$ phase diagrams, while the other calculations are for (arbitrary) fixed temperature. This means that we vary $b$ at constant $\xi$ and constant $R$. Notice further that the GinzburgLandau parameter $\kappa=\lambda / \xi$ is independent of the temperature.

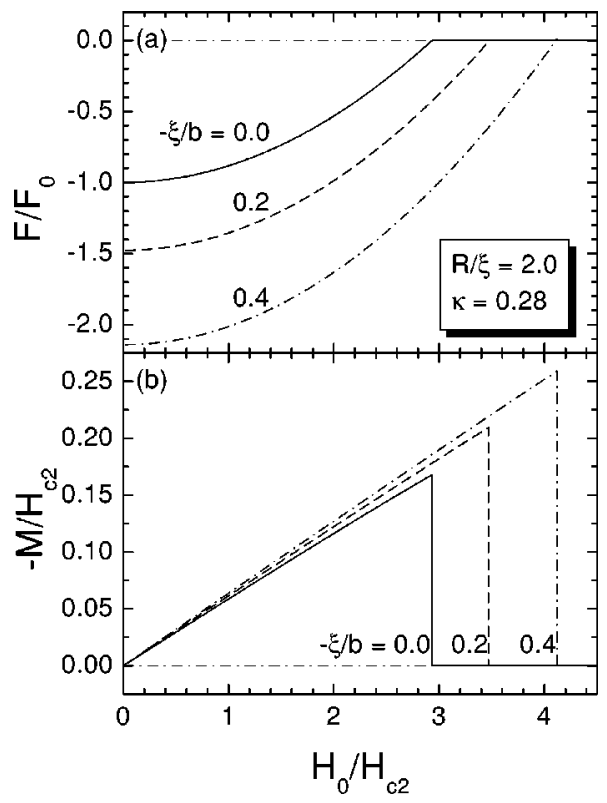

FIG. 1. (a) The ground state free energy and (b) the magnetization as a function of the applied magnetic field for a superconducting cylinder with radius $R=2.0 \xi$ for $\kappa=0.28$ and $-\xi / b=0$ (solid curves), 0.2 (dashed curves), and 0.4 (dash-dotted curves).

\section{SMALL CYLINDERS}

First we discuss infinitely long cylinders with a small radius, where we may limit ourselves to the giant vortex states. In this case the confinement effects are dominant and they impose the cylindrical symmetry of the boundary of the cylinder on the vortex state. Consequently, the dimensions of the Ginzburg-Landau equations are reduced, which improves the accuracy and the computation time.

\section{A. Type-I cylinders}

We consider infinite long cylinders with radius $R=2.0 \xi$ for a Ginzburg-Landau parameter $\kappa=0.28$. Figures 1 (a), 1 (b) show the ground state free energy, i.e., the thermodynamic equilibrium free energy, and the magnetization as a function of the applied magnetic field for such a superconducting cylinder for the usual boundary condition, i.e., $-\xi / b=0.0$ (solid curves), and for $-\xi / b=0.2$ (dashed curves) and 0.4 (dash-dotted curves), which correspond to surface enhancement. With increasing $-\xi / b$ the free energy at zero magnetic field becomes more negative, which means an enhancement of superconductivity. Also the ground state superconducting/ normal transition moves to higher fields. Notice that the magnetic field expulsion [see Fig. 1(b)] is enhanced with increasing $-\xi / b$.

Figures 2(a)-2(c) show the radial dependence of the Cooper-pair density, the magnetic field, and the current density, respectively, for the previously considered cylinder at the applied magnetic field $H_{0} / H_{c 2}=2.02$ and for vorticity $L=0$. The solid curves give the result for $-\xi / b=0$, the dashed curves for $-\xi / b=0.2$ and the dash-dotted curves for $-\xi / b=0.4$. From Fig. 2(a) it is very clear that the Cooperpair density near the surface, and hence the surface super- 


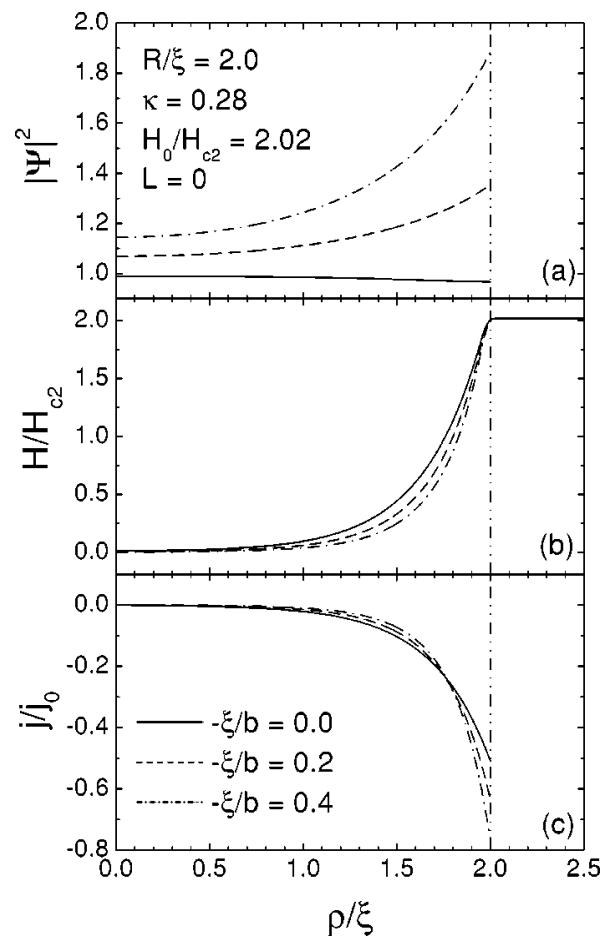

FIG. 2. (a) The Cooper-pair density, (b) the magnetic field, and (c) the current density as a function of the radial position for a cylinder with radius $R=2.0 \xi$ and for $\kappa=0.28$. The external field is $H_{0} / H_{c 2}=2.02$ and the vorticity is $L=0$.

conductivity, increases with increasing $-\xi / b$. For a small cylinder radius, also the Cooper-pair density in the center of the cylinder is influenced by the boundary condition. From Fig. 2(b) one can see that enhancing the surface superconductivity results in a more pronounced Meissner effect, i.e., the magnetic field expulsion becomes more complete with increasing $-\xi / b$. Notice that the magnetic field for this situation is always zero in the center of the cylinder. To expel the magnetic field more, the superconductor has to induce more superconducting current near the surface. Figure 2(c) shows that the current density is zero in the center of the superconductor and becomes more negative near the surface. The superconducting Meissner currents are also more concentrated near the surface with increasing $-\xi / b$. For the Meissner state the current has the same sign in the whole sample and is thus flowing in the whole sample in the same direction.

In Fig. 3 the dependence of the transition fields for the different giant vortex states on the surface enhancement $b$ is given for a superconducting cylinder with radius $R=2.0 \xi$ and $\kappa=0.28$. The solid curves give the ground state transitions between the different $L$ states and the thick solid curve indicates the superconducting/normal transition. For the normal boundary condition $-\xi / b=0$ it is evident that the ground state consists of the Meissner state, i.e., $L=0$, since $\kappa$ is sufficiently small. The superconducting/normal transition occurs at $H_{0} / H_{c 2} \approx 2.9$. What happens with increasing $-\xi / b$ ? (i) For $-\xi / b<0.65$ the ground state is still given by the Meissner state, and with increasing $-\xi / b$ the superconducting/normal-transition moves to higher fields. (ii) For $-\xi / b>0.65$ different $L$ states become the ground

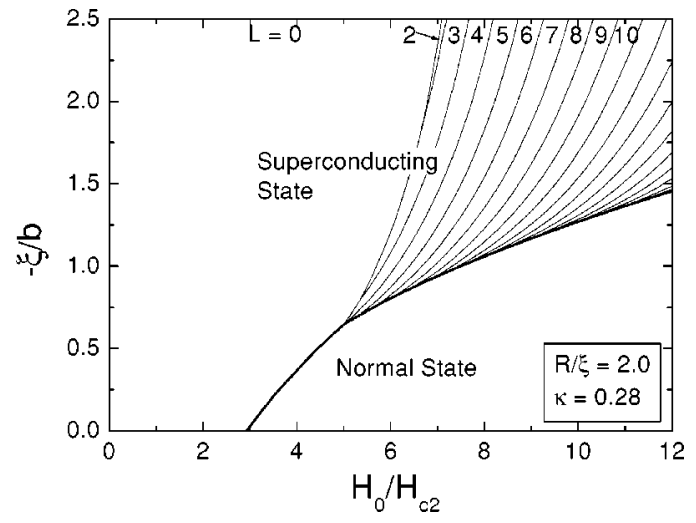

FIG. 3. The $(-\xi / b)-H$ phase diagram for a superconducting cylinder with radius $R=2.0 \xi$ and $\kappa=0.28$. The solid curves indicate the transitions between the different $L$ states and the thick curve gives the superconducting/normal transition.

state. This is in agreement with the results of Montevecchi ${ }^{28}$ who also found transitions between different $L$ states for $b<0$ for a fixed cylinder radius $R /|b|=1.2$ and $\kappa=0.3$ (see Fig. 1.14 of Ref. 28). Remarkably we find that the ground state does not evolve from the Meissner state to a state with vorticity $L=1$. Rather it evolves to a state with larger vorticity. For $0.65 \leqq-\xi / b \leqq 0.8$ the ground state changes from the Meissner state to a state with $L=4$, for $0.8 \lesssim-\xi / b$ $\lesssim 1.8$ to a state with $L=3$, and for $-\xi / b \gtrsim 1.8$ to a state with $L=2$. (iii) Further increasing $-\xi / b$ the ground state will change first from $L=0$ to $L=1$. The latter transition corresponds to type-II behavior which only occurs in the absence of surface enhancement for $\kappa$ larger than some critical Ginzburg-Landau parameter $\kappa_{2}$.

The three different regimes which we found for the case of increasing $-\xi / b$ for fixed small $\kappa$ are very similar to the three regimes for the case of increasing $\kappa$ for the normal boundary condition, i.e., $-\xi / b=0$. (i) For the normal boundary condition it is known ${ }^{29}$ that only the Meissner state is the ground state for $\kappa$ smaller than a critical parameter $\kappa_{1}$. (ii) For $\kappa_{1} \lesssim \kappa \leqslant \kappa_{2}$ surface superconductivity can occur and states with vorticity larger than one exist. For example, Fink and Presson ${ }^{19}$ found ground state transitions from the Meissner state with $L=0$ into surface superconducting states with $L=4$ for a superconducting cylinder with radius $R=3.0 \xi$ and $\kappa=0.5$. (iii) For $\kappa>\kappa_{2}$ (type II superconductivity) the ground state transits from the Meissner state to states with $L=1,2, \ldots, n$, successively, where the value of $n$ depends on the radius and the Ginzburg-Landau parameter (see, for example, Ref. 19). In bulk superconductors $\kappa_{1} \approx 0.42$ and $\kappa_{2}$ $=1 / \sqrt{2}$, but for cylinders these parameters are radius dependent. $^{21}$

What is the reason for this remarkable behavior of the ground state for increasing $-\xi / b$ ? (i) For $-\xi / b<0.65$ the order parameter, and thus the Cooper-pair density, near the cylinder boundary increases with increasing $-\xi / b$ which also leads to an enhancement of the order parameter in the center, albeit less pronounced [see also Fig. 2(a)]. Consequently, the free energy at zero magnetic field becomes more negative and the superconducting/normal transition moves to 


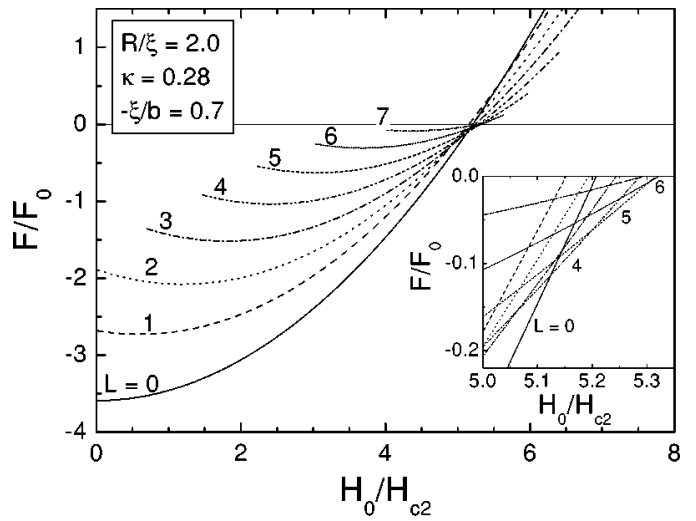

FIG. 4. The free energy for a superconducting cylinder with radius $R / \xi=2.0$ and $\kappa=0.28$ for $-\xi / b=0.7$ as a function of the external magnetic field.

higher fields [see also Fig. 1(a)]. (ii) With increasing $-\xi / b$ the Cooper-pair density in the center for the $L=0$ state is not increasing as strongly as the Cooper-pair density near the boundary and the cylinder remains superconducting at higher fields. For $-\xi / b \gtrsim 0.65$ it becomes energetically less favorable to expell rather high fluxes from the sample. The effect of the increase of vorticity on the free energy is shown in Fig. 4 for $-\xi / b=0.7$. From the inset it is clear that the free energy of the $L=4$ state becomes lower than the one of the Meissner state at $H_{0} / H_{c 2} \approx 5.12$. This means that for this field the penetration of four fluxes becomes more favorable than the expulsion of the field. At $H_{0} / H_{c 2} \approx 5.20,5.30$, and 5.32 the ground state changes, respectively, into the $L=5$ state, the $L=6$ state, and the normal state. Further increasing $-\xi / b$ leads to higher Cooper-pair density near the boundary and, consequently, to higher induced supercurrents. As a result, a smaller amount of flux will penetrate into the sample after the first ground state transition. For example, with increasing field at $-\xi / b>0.8$ the ground state changes from the Meissner state into the $L=3$ state and at higher fields into states with $L=4,5,6$ and so on. (iii) For high values of $-\xi / b$, the explanation is analogous as above but now the ground state changes from $L=0$ to $L=1,2,3$, and so forth.

In Fig. 5 we plot the $H-T$ phase diagram for a supercon-

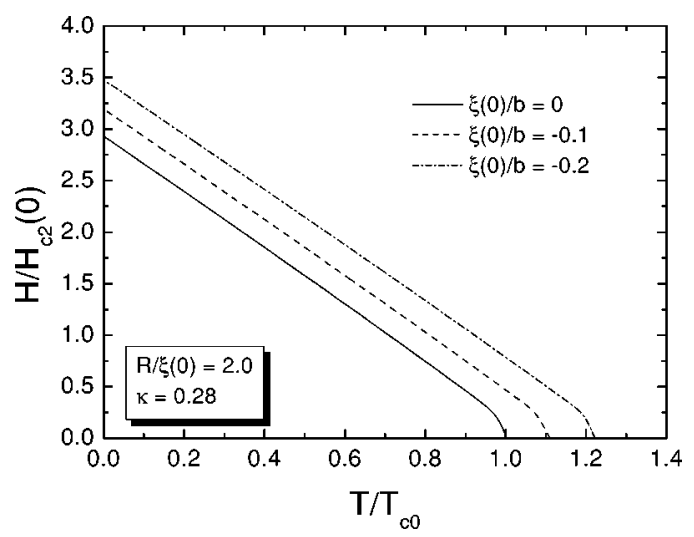

FIG. 5. The $H-T$ phase diagram for a superconducting cylinder with radius $R=2.0 \xi$ and $\kappa=0.28$ for $-\xi / b=0.0$ (solid curve), 0.2 (dashed curve), and 0.4 (dash-dotted curve).

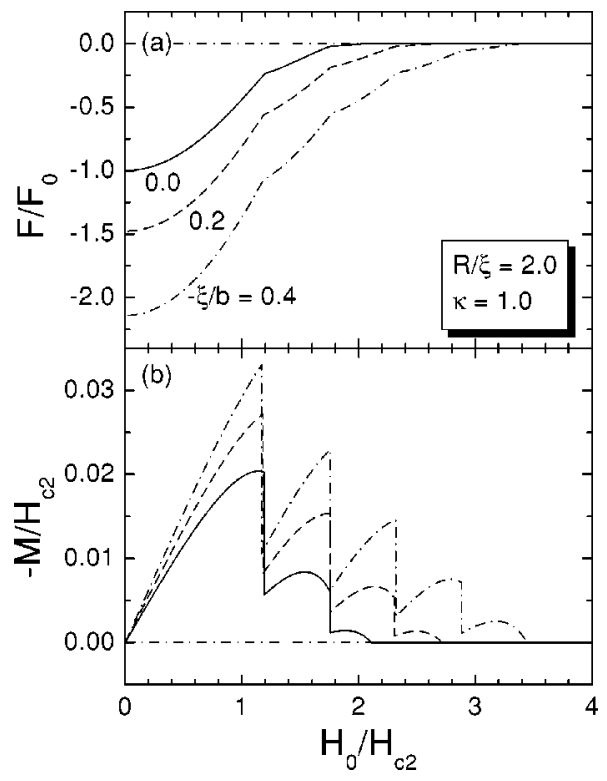

FIG. 6. (a) The ground state free energy and (b) the magnetization as a function of the applied magnetic field for a superconducting cylinder with radius $R=2.0 \xi$ for $\kappa=1.0$ and $-\xi / b=0$ (solid curves), 0.2 (dashed curves), and 0.4 (dash-dotted curves).

ducting cylinder with $R=2.0 \xi$ and $\kappa=0.28$ for $-\xi(0) / b$ $=0.0,0.1$, and 0.2 . For these values of $-\xi(0) / b$ the superconducting ground state is always given by the Meissner state (see also Fig. 3). The superconducting/normal transition is shown by a solid curve for $-\xi(0) / b=0.0$, by a dashed curve for $-\xi(0) / b=0.1$, and by a dash-dotted curve for $-\xi(0) / b=0.2$. For $\kappa=0$ the superconducting/normal transition is a straight line in the $H-T$ phase diagram. For $\kappa$ $=0.28$, the superconducting/normal transition is still given by a straight line for $T \ll T_{c}$, regardless of the value of $-\xi(0) / b$, but it has now a curvature near $T_{c}$. With increasing $-\xi(0) / b$ the superconducting/normal transition, which is of first order, moves to higher temperatures for fixed field or to higher fields for fixed temperature.

\section{B. Type-II cylinders}

We consider infinite cylinders with radius $R=2.0 \xi$ for a Ginzburg-Landau parameter $\kappa=1.0$. Figures 6(a), 6(b) show the ground state free energy and magnetization as a function of the applied magnetic field for such a superconducting cylinder for $-\xi / b=0.0$ (solid curves), 0.2 (dashed curves), and 0.4 (dash-dotted curves). Now, the superconducting ground state is not always the Meissner state. For example, for the usual boundary condition, i.e., $-\xi / b=0.0$, the vorticity of the ground state is $L=0$ for $H_{0} / H_{c 2} \leqslant 1.195$. At the first transition field $H_{0} / H_{c 2}=1.195$ the vorticity of the ground state changes from $L=0$ to $L=1$ and then it remains $L=1$ until $H_{0} / H_{c 2}=1.7575$ where it changes to $L=2$. At $H_{0} / H_{c 2}=2.12$ the free energy of the ground state becomes zero and the superconducting/normal transition takes place. The transitions between the different $L$ states are indicated by corners in the free energy and jumps in the magnetization. As for type-I cylinders, the free energy at $H_{0}=0$ becomes 


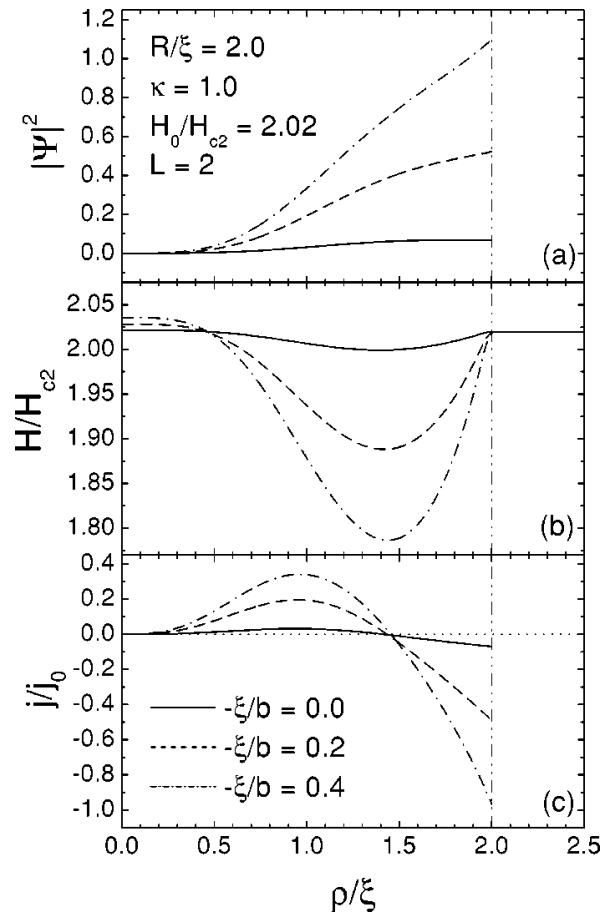

FIG. 7. (a) The Cooper-pair density, (b) the magnetic field, and (c) the current density as a function of the radial position for a cylinder with radius $R=2.0 \xi$ and for $\kappa=1.0$. The external field is $H_{0} / H_{c 2}=2.02$ and the vorticity is $L=2$.

more negative with increasing $-\xi / b$, i.e., superconductivity is enhanced, and the transition to the normal state moves to higher fields. Furthermore, the vorticity of the ground state can become larger and the peaks in the magnetization are higher, indicating a more efficient expulsion of the magnetic field from the superconductor. Notice that the magnetic fields for the transitions between different $L$ states are almost independent of the value of $-\xi / b$, but the surface critical field $H_{c 3}$ is a sensitive function of $-\xi / b$.

Next, we investigate the radial dependence of the Cooperpair density, the magnetic field and the current density for such a superconducting cylinder. For vorticity $L=0$ the results are analogous to the results for type-I cylinders which were described in Fig. 2. In Figs. 7(a)-7(c) we show the radial dependence of, respectively, the Cooper-pair density, the magnetic field and the current density for the ground state at $H_{0} / H_{c 2}=2.02$, i.e., the giant vortex state with vorticity $L=2$. The solid curves give the result for $-\xi / b=0$, the dashed curves for $-\xi / b=0.2$, and the dash-dotted curves for $-\xi / b=0.4$. In Fig. 7(a) it is shown that the Cooper-pair density is zero for all $-\xi / b$ in the center of the cylinder, i.e., at the position of the giant vortex with vorticity $L=2$. Near the surface the Cooper-pair density is enhanced, which is understandable because the slope of $|\Psi|$ at $\rho=R$ is given by $-\xi / b$. For the giant vortex state, the magnetic field is not zero in the center, as was the case for $L=0$, but it can be even higher than the external field $H_{0}=2.02 H_{c 2}$. With increasing $-\xi / b$, the superconductivity near the boundary is enhanced and, thus, more magnetic field can be expelled from the cylinder and the giant vortex will be more compressed in the center [see Fig. 7(b)]. As a consequence, the

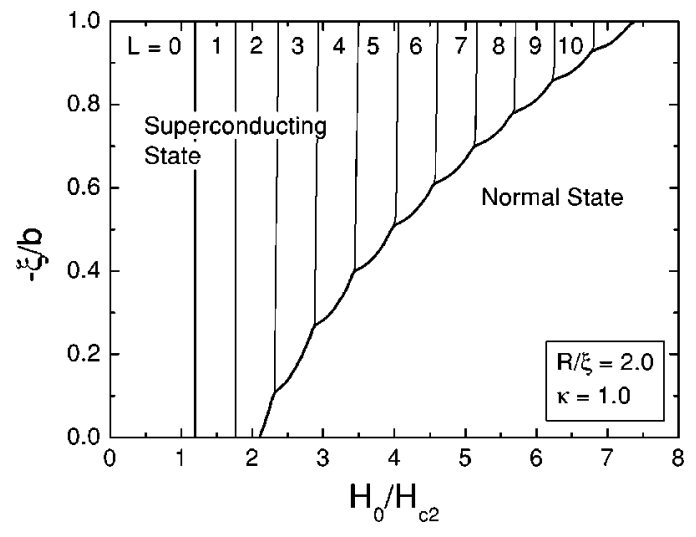

FIG. 8. The $(-\xi / b)-H$ phase diagram for a superconducting cylinder with radius $R=2.0 \xi$ and $\kappa=1.0$. The solid curves indicate the transitions between the different $L$ states and the thick curve gives the superconducting/normal transition.

minimum of magnetic field inside the cylinder decreases and the magnetic field at the position of the vortex increases. To expel the magnetic field more and to compress the giant vortex better in the center, the superconductor has to induce a larger superconducting current. Therefore the superconducting current is more positive close to the giant vortex and more negative near the boundary [see Fig. 7(c)]. Notice that also for giant vortex states there is no qualitative difference between the radial dependences of the Cooper-pair density, the magnetic field and the current density for type-I and type-II superconductors. For a giant vortex state with vorticity $L$ always the same amount of flux has to be trapped in the center of the cylinder. Of course, the magnetic field region where the giant vortex state with vorticity $L$ nucleates does depend strongly on $\kappa$. Notice further that for low $\kappa$ these giant vortex states are not ground states, but are metastable states.

The fact that the transition fields between different $L$ states are almost independent of the value of $-\xi / b$, can also be seen from the $(-\xi / b)-H$ phase diagram for a superconductor cylinder with radius $R=2.0 \xi$ and $\kappa=1.0$, which is shown in Fig. 8. The thin solid curves give the ground state transitions between the different $L$ states and the thick solid curve indicates the superconducting/normal transition. For $\kappa=1.0$ we do not find ground state transitions between a state with vorticity $L=0$ and vorticity $L>1$, as was the case for $\kappa=0.28$. Moreover, the magnetic field range over which the ground state has a particular vorticity $L$ is almost the same for all $L>0$, namely, $\Delta H_{L}=H_{L \rightarrow L+1}-H_{L-1 \rightarrow L}$ $\approx 0.57 H_{c 2}$. Figure 7 indicates that the width of the superconducting sheath in $\rho / \xi$ is independent of $b$. The value of $b$ influences only the amplitude of the order parameter near the surface [Fig. 7(a)], the diamagnetization [Fig. 7(b)], and the strength of the currents [Fig. 7(c)]. Therefore, the space available for the confined flux in the core region of the cylinder does (almost) not depend on the enhancement. As a consequence, the number of fluxoids, or vorticity $L$, depends only on the strength of the external field $H_{0}$ and almost not on $b$ for fixed temperature. 


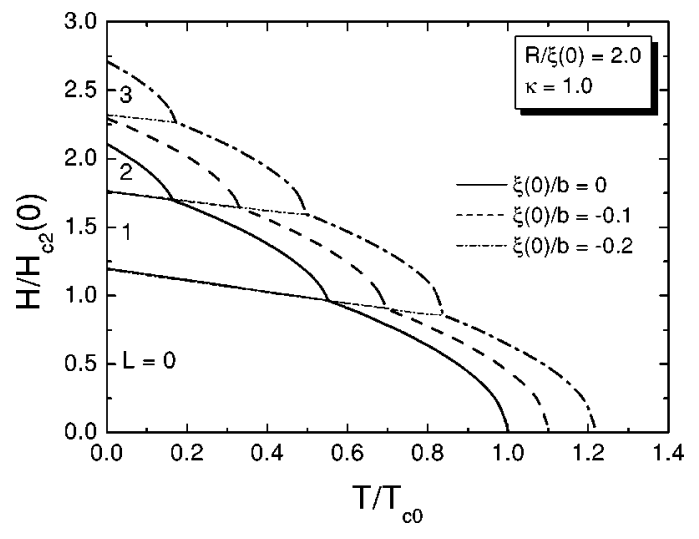

FIG. 9. The $H-T$ phase diagram for a superconducting cylinder with radius $R=2.0 \xi$ and $\kappa=1.0$ for $-\xi / b=0.0$ (solid curves), 0.2 (dashed curves), and 0.4 (dash-dotted curves). The thick curves indicate the superconducting/normal transition and the thinner curves the ground state transitions between the giant vortex states with different vorticity $L$.

In Fig. 9 we plot the $H-T$ phase diagram for a superconducting cylinder with $R=2.0 \xi$ and $\kappa=1.0$ for $-\xi(0) / b$ $=0.0,0.1$, and 0.2 . The ground state transitions are shown by solid curves for $-\xi(0) / b=0.0$, by dashed curves for $-\xi(0) / b=0.1$, and by dash-dotted curves for $-\xi(0) / b$ $=0.2$. The thick curves indicate the superconducting/normal transition and the thinner curves (they are almost straight lines) show the ground state transitions between the giant vortex states $L \leftrightarrow L+1$. From Fig. 9 it is clear that the transitions between the different $L$ states are (almost) independent of the value of $-\xi / b$, but the superconducting/normal transition is a sensitive function of $-\xi / b$ (see also Fig. 8). With increasing $-\xi(0) / b$ the superconducting/normal transition moves to higher temperatures for fixed field or to higher fields for fixed temperature as was also the case for $\kappa=0.28$. The zero field critical temperature is the same for $\kappa=1.0$ as for $\kappa=0.28$ and depends only on $-\xi(0) / b$. The critical fields at zero temperature are different in both cases. For $-\xi(0) / b=0.2$, for example, $H_{c 3}(0)=3.5 H_{c 2}(0)$ for $\kappa$ $=0.28$ and $H_{c 3}(0)=2.7 H_{c 2}(0)$ for $\kappa=1.0$. In fact, the "zero" temperature corresponds to some reference temperature rather than the real temperature because the GinzburgLandau theory is not valid at very low temperatures. Another significant difference is that for $\kappa=1.0$ the superconducting/ normal transition is no longer a straight line for temperatures $T \ll T_{c}$, but consists of corners which indicate the transition between the different $L$ states. These corners are bicritical points where three phases become identical for a given value of $-\xi(0) / b$. For example, the normal state coincides with the $L=0$ state and the $L=1$ state at $H_{0} / H_{c 2}(0)=0.86$ and $T / T_{c 0}=0.84$ for $-\xi(0) / b=0.2$. Note that all transitions between different $L$ states are of first order and correspond to free energy crossings, while the superconducting/normal transition is a second order transition. These bicritical points occur at magnetic fields which are only weakly sensitive to the surface enhancement, while the transition temperatures are quite sensitive to $b$. Notice further that the superconducting/normal phase transition boundary is exactly the same for a cylinder with $\kappa=1.0$ as for a very thin disk (thickness $d \ll \xi$ ) with the same radius, if the same boundary condition [Eq. (2b)] is taken on the sides, but the standard boundary condition $(-\xi / b=0)$ on the top and the bottom of the disk. This leads to a $z$-independent order parameter and, as a consequence, to similar physics as for an infinitely long cylinder. This can be seen by comparing Fig. 9 with Fig. 14 of Ref. 25. In the case of the disk, the field is perpendicular to the disk.

\section{LARGE CYLINDERS: MULTIVORTICES}

Up to now, we considered only cylinders with small radii where the confinement effects were dominant and only giant vortex states were stable. Now, we consider superconducting cylinders with a large radius in which multivortex states can nucleate for certain magnetic fields. There exists a crossover radius $R_{c}$ such that for $R<R_{c}$ only giant vortex states can nucleate, while for $R \geqslant R_{c}$ multivortex states can nucleate for a certain vorticity and magnetic field. The crossover radius $R_{c}$ depends strongly on the Ginzburg-Landau parameter $\kappa$ and on $-\xi / b$, and can only be determined numerically. For example, $R_{c} \approx 2.8$ for $\kappa=1.0$ and $-\xi / b=0$, which becomes $R_{c} \approx 3.0$ for $-\xi / b=0.4$ and $\kappa=1.0$. This means that we can no longer limit our calculations to axially symmetric solutions. Nevertheless, from the study of $\operatorname{disks}^{6}$ and rings ${ }^{14}$ we know that the transition fields between states with different vorticity do (almost) not depend on the fact whether one considers axial symmetry or not. Therefore, we can still calculate the $(-\xi / b)-H$ phase diagrams using the cylindrical symmetry approach, but if we want to know the real vortex configurations, we have to use the general theory in order to account for multivortices.

We calculate the $(-\xi / b)-H$ phase diagrams for a superconducting cylinder with radius $R=4.0 \xi$ for $\kappa=0.28$ and $\kappa$ $=1.0$, which are shown in Figs. 10(a), 10(b), respectively. The thin solid curves give the ground state transitions between the different $L$ states and the thick solid curve indicates the superconducting/normal transition. The conclusions are similar to those for small cylinders, but with many more transitions between different $L$ states. However, for $\kappa$ $=0.28$ we find now transitions from the Meissner state immediately to a state with vorticity $L=13$ as a function of the magnetic field in the range $0.43 \leqslant-\xi / b \leqslant 0.47$. With further increasing $-\xi / b$ we find first transitions from $L=0$ to $L$ $=12,11,10$, and 9, respectively. Notice that triple points occur where the Meissner state coexists with two different giant vortex states. For example, at $H_{0} / H_{c 2} \approx 3.4$ and $-\xi / b \approx 0.47$ the Meissner state coexists with the $L=12$ state and the $L=13$ state. This means that these three states have the same free energy in that case.

For $\kappa=1.0$ the magnetic field range over which the ground state has a particular vorticity $L$ is almost the same for all $L>0$, namely $\Delta H_{L} \approx 0.125 H_{c 2}$, which means that the ground state changes more quickly its vorticity $L$ than for cylinders with radius $R=2.0 \xi$, where $\Delta H_{L} \approx 0.57 H_{c 2}$, which is due to the larger cross section of the cylinder.

Next we will compare the free energy and the magnetization for the usual boundary condition $-\xi / b=0$ with the case 

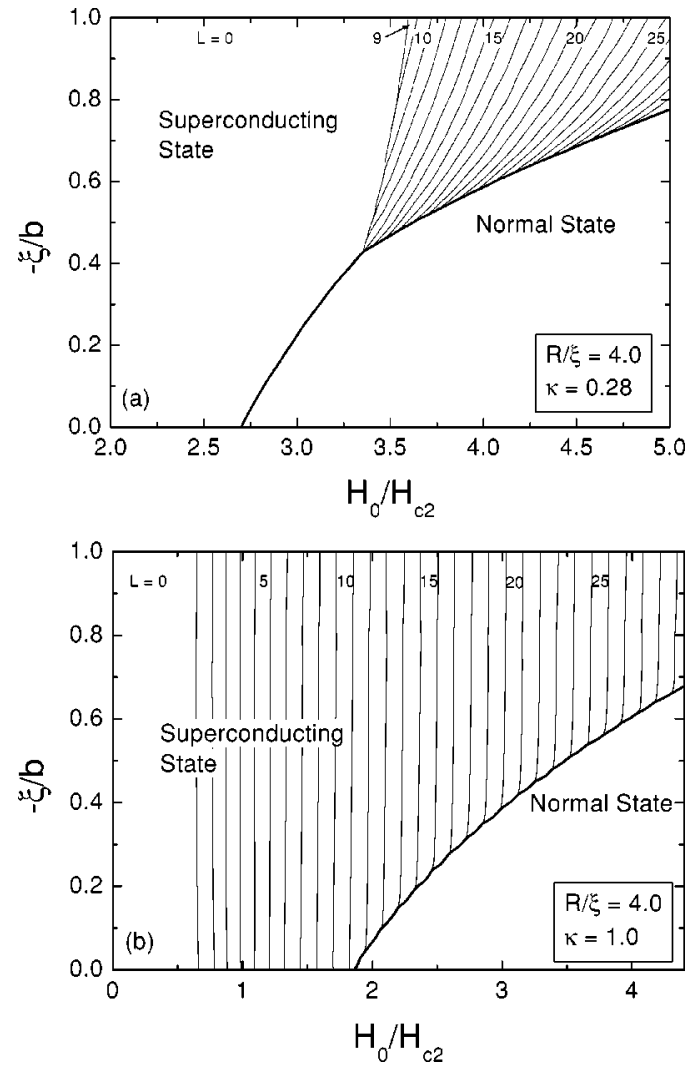

FIG. 10. The $(-\xi / b)-H$ phase diagram for a superconducting cylinder with radius $R=4.0 \xi$ for $\kappa=0.28$ (a) and $\kappa=1.0$ (b). The solid curves indicate the transitions between the different $L$ states and the thick curve gives the superconducting/normal transition.

of surface enhancement, $-\xi / b=0.2$. Figures 11(a), 11(b) show the free energy and the magnetization for a cylinder with radius $R=4.0 \xi$ and $\kappa=1.0$ for the usual boundary condition. The different giant vortex states are indicated by the solid curves, the multivortex states by the dashed curves and the multivortex to giant vortex transition fields $H_{M G}$ by the open circles. Notice that we find superconducting states up to vorticity $L=11$ and that the superconducting/normal transition field is $H_{c 3} / H_{c 2} \approx 1.85$ [see also Fig. 10(b)]. Multivortices can nucleate for vorticities $L=2,3$, and 4 . The multivortex state with $L=2$ can nucleate for $0.295 \leqslant H_{0} / H_{c 2}$ $\leqslant 0.92$, with $L=3$ for $0.42 \leqslant H_{0} / H_{c 2} \leqslant 1.045$, and with $L$ $=4$ for $0.5325 \leqslant H_{0} / H_{c 2} \leqslant 0.92$. In Fig. 11(b) the equilibrium ground state transitions are indicated by the vertical curves.

Figures 12(a), 12(b) show the free energy and the magnetization for a cylinder with radius $R=4.0 \xi$ and $\kappa=1.0$ for $-\xi / b=0.2$. The different giant vortex states are indicated by the solid curves, the multivortex states by the dashed curves and the multivortex to giant vortex transition fields $H_{M G}$ by the open circles. The latter transition is of second order. For this boundary condition the superconducting/normal transition field is $H_{c 3} / H_{c 2} \approx 2.35$ which is appreciably higher than for $-\xi / b=0$. Also vortex states with higher vorticity are stable, up to 15 instead of up to 11 for $-\xi / b=0$. Multivortex states can nucleate with $L=2$ for $0.1325 \leqslant H_{0} / H_{c 2} \leqslant 0.945$,

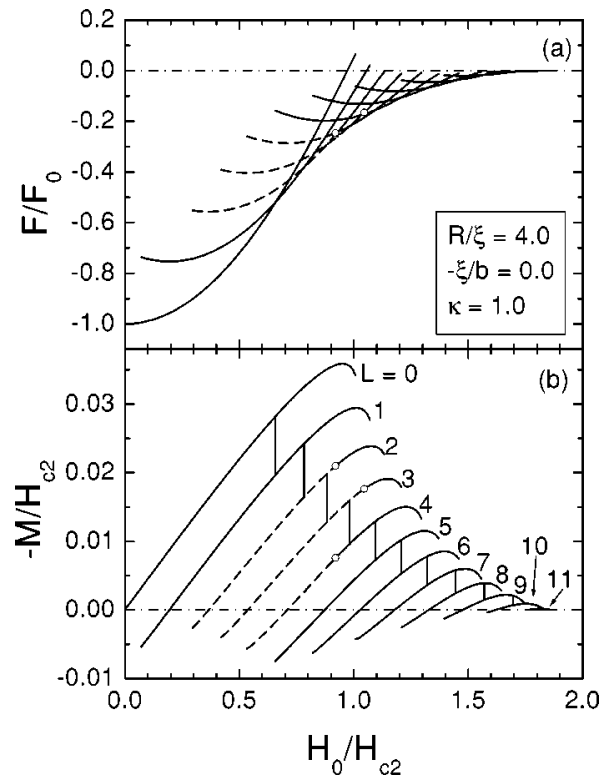

FIG. 11. (a) Free energy and (b) magnetization for vortex states in a superconducting cylinder with radius $R=4.0 \xi$ and $\kappa=1.0$ for the normal boundary condition $-\xi / b=0$. Giant vortex states are given by solid curves, multivortex states by dashed curves, and the open circles indicate the multivortex to giant vortex transition fields. The vertical lines in (b) give the ground state transitions.

with $L=3$ for $0.2575 \leqslant H_{0} / H_{c 2} \leqslant 1.02$, and with $L=4$ for $0.3825 \leqslant H_{0} / H_{c 2} \leqslant 0.895$.

How does the value of $-\xi / b$ influence the nucleation of the multivortex states? Figure 13 shows the free energy of the $L=3$ state for a superconducting cylinder with radius $R$

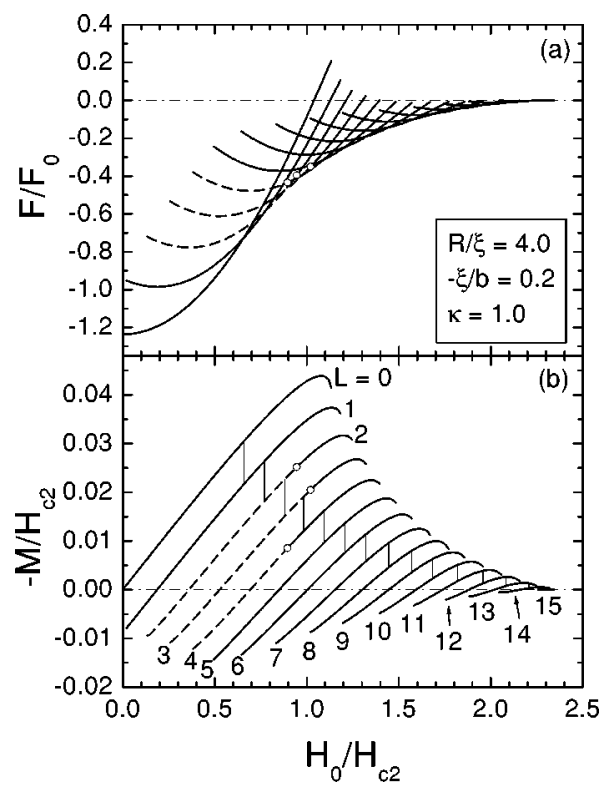

FIG. 12. (a) Free energy and (b) magnetization for vortex states in a superconducting cylinder with radius $R=4.0 \xi$ and $\kappa=1.0$ for the boundary condition $-\xi / b=0.2$. Giant vortex states are given by solid curves, multivortex states by dashed curves, and the open circles indicate the multivortex to giant vortex transition fields. The vertical lines in (b) give the ground state transitions. 


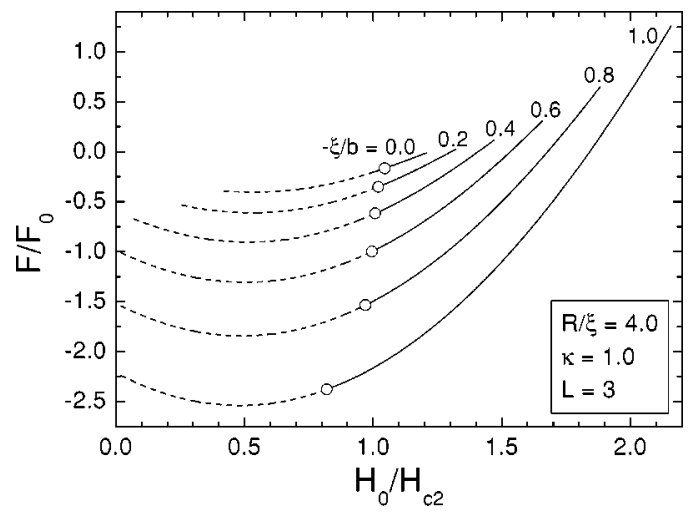

FIG. 13. Free energy for the $L=3$ state in a superconducting cylinder with radius $R=4.0 \xi$ and $\kappa=1.0$ for $-\xi / b=0.0,0.2,0.4$, $0.6,0.8$, and 1.0. Giant vortex states are given by solid curves, multivortex states by dashed curves and the open circles indicate the multivortex to giant vortex transition fields.

$=4.0 \xi$ and $\kappa=1.0$ for $-\xi / b=0.0,0.2,0.4,0.6,0.8$, and 1.0 as a function of the applied magnetic field. Giant vortex states are given by solid curves and multivortex states by dashed curves. The transitions from a multivortex state to a giant vortex state are indicated by the open circles. With increasing $-\xi / b$ the $L=3$ state can nucleate up to larger fields, i.e., $H_{\text {nuc }} / H_{c 2}=1.21,1.32,1.47,1.66,1.88$, and 2.16 for $-\xi / b=0.0,0.2,0.4,0.6,0.8$, and 1.0 , respectively. On the other hand the multivortex to giant vortex transition field decreases, i.e., $H_{M G} / H_{c 2}=1.045,1.02,1.0075,0.995,0.97$, and 0.82 for $-\xi / b=0.0,0.2,0.4,0.6,0.8$, and 1.0 , respectively. This means that with increasing $-\xi / b$ the magnetic field region over which the giant vortex state exists increases and the one of the multivortex state decreases. The reason is that increasing $-\xi / b$ corresponds to an enhancement of superconductivity near the boundary. Therefore, it is more difficult for the magnetic field to penetrate near the cylinder edge than in the center. Since multivortices are situated more closely to the cylinder edge, it is obvious that multivortices become unstable at lower fields for increasing $-\xi / b$. Or alternatively, the confinement effects become more important for increasing surface superconductivity.

To illustrate this better, we show the Cooper-pair density of the $L=3$ state for such a cylinder in Figs. 14(a)-14(f) at $H_{0} / H_{c 2}=0.445$ for $-\xi / b=0.0,0.2,0.4,0.6,0.8$, and 1.0 , respectively. High Cooper-pair density is given by dark regions, low Cooper-pair density by light regions. The three vortices correspond to the three white spots. For the usual boundary condition $-\xi / b=0.0$ the vortices are clearly separated. With increasing $-\xi / b$ the vortices are pushed to the center and at $-\xi / b=0.4$ they already start to overlap. For $-\xi / b=0.8$ and 1.0 the vortices are very close to each other. Therefore, we plotted the logarithm of the Cooper-pair density to show the positions of the vortices and to prove that it it still a multivortex state. Thus, by the enhancement of the Cooper-pair density near the boundary the vortices move to the center and for increasing $-\xi / b$ they will recombine in the center creating a giant vortex state.

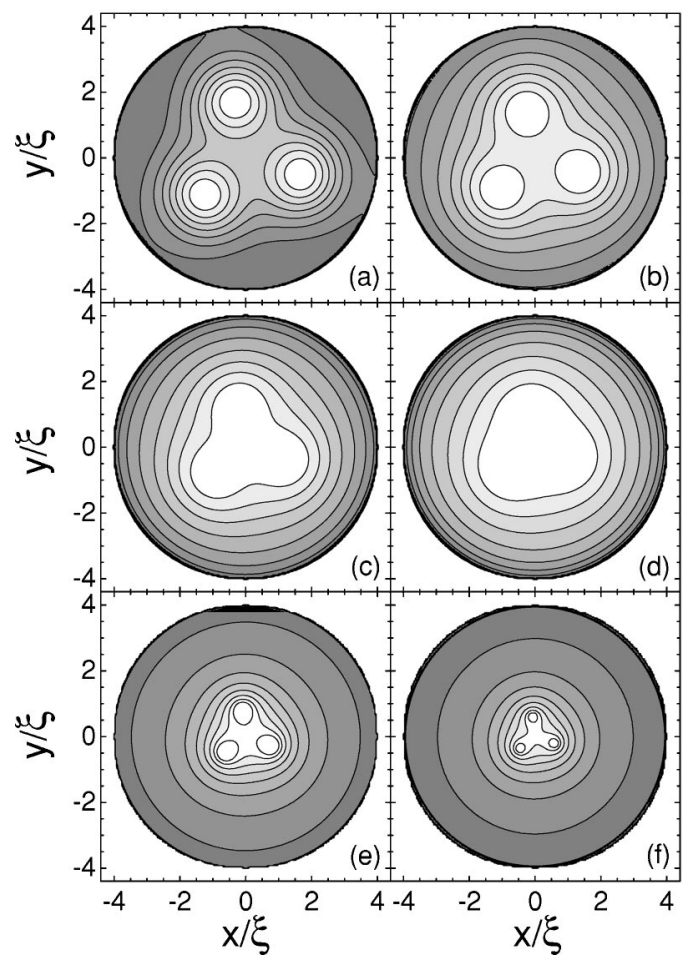

FIG. 14. The Cooper-pair density for the multivortex state with $L=3$ in a cylinder with $R / \xi=4.0$ and $\kappa=1.0$ at $H_{0} / H_{c 2}=0.445$ for $-\xi / b=0.0$ (a), 0.2 (b), 0.4 (c), 0.6 (d), and the logarithm of the Cooper-pair density for $-\xi / b=0.8$ (e) and 1.0 (f).

\section{CONCLUSIONS}

In the present paper we investigated the effect of the enhancement of surface superconductivity on the critical field and the critical temperature for superconducting cylinders with radii comparable to the coherence length $\xi$. We also studied the influence of a the Ginzburg-Landau parameter $\kappa$. A distinction was made between cylinders with small radii where the confinement effects dominate and only giant vortex states exist, and cylinders with a larger radius where multivortices can nucleate for certain magnetic fields and vorticities.

Generally, increasing $-\xi / b$ leads to a more negative free energy at $H=0$ and to a higher superconducting/normal transition field. We also studied the magnetic field distribution, the Cooper-pair density and the current density for different values of the surface enhancement. For higher values of $-\xi / b$ more magnetic field can be expelled from the cylinder and the giant vortex in the center is compressed more. Therefore, higher currents are induced near the boundary and near the giant vortex. The Cooper-pair density close to the boundary increases, and for small cylinders this also influences the Cooper-pair density in the center.

From the $H-T$ phase diagrams we found that the critical temperature depends on $-\xi / b$, while it is independent of $\kappa$ (see Ref. 24). With increasing $-\xi / b$ the critical temperature increases for fixed magnetic field and the critical magnetic field increases for fixed temperature.

We also obtained $(-\xi / b)-H$ phase diagrams. In type-I cylinders the surface enhancement has drastic consequences. 
Even at low $\kappa$ the surface enhancement leads to transitions between different $L$ states and thus to type-II behavior. Moreover, as a function of the magnetic field the superconducting ground state transits from the Meissner state to a vortex state with $L>1$ over a range of $-\xi / b$ values. Therefore, we can conclude that increasing $-\xi / b$ for type-I superconductors seems to have a similar effect as increasing $\kappa$ for fixed $-\xi / b$ for certain properties of the superconducting cylinder. In type-II cylinders we found that the magnetic field range over which the ground state has a particular vorticity $L$ is almost the same for all $L>1$. This magnetic field range decreases with increasing cylinder radius.

If the cylinder radius is sufficiently large, multivortex states can nucleate and we studied the influence of the sur- face enhancement on the nucleation of these states. We found that at fixed field and with increasing $-\xi / b$ the multivortices move to the center creating a giant vortex state. Thus surface enhancement destabilizes the multivortex state.

\section{ACKNOWLEDGMENTS}

This work was supported by the Flemish Science Foundation (FWO-Vl) Project No. G.0277.97N, the Flemish Concerted Action (GOA), the Inter-University Attraction Poles (IUAP) research programs, and the European ESF-Vortex Matter. Discussions with G. F. Zharkov are gratefully acknowledged. We are indebted to the late Dr. V. A. Schweigert for help in the initial stage of this work.
*Permanent address: Donetsk Physical \& Technical Institute, National Academy of Sciences of Ukraine, Donetsk 83114, Ukraine.

†Electronic address: peeters@uia.ua.ac.be

${ }^{1}$ O. Buisson, P. Gandit, R. Rammel, Y.Y. Wang, and B. Pannetier, Phys. Lett. A 150, 36 (1990).

${ }^{2}$ P.S. Deo, V.A. Schweigert, F.M. Peeters, and A.K. Geim, Phys. Rev. Lett. 79, 4653 (1997).

${ }^{3}$ R. Benoist and W. Zwerger, Z. Phys. B 103, 377 (1997).

${ }^{4}$ V.A. Schweigert and F.M. Peeters, Phys. Rev. B 57, 13817 (1998)

${ }^{5}$ A.K. Geim, I.V. Grigorieva, S.V. Dubonos, J.G.S. Lok, J.C. Maan, A.E. Filippov, and F.M. Peeters, Nature (London) 390, 256 (1997).

${ }^{6}$ V.A. Schweigert, F.M. Peeters, and P.S. Deo, Phys. Rev. Lett. 81, 2783 (1998).

${ }^{7}$ J.J. Palacios, Physica B 256-258, 610 (1998); Phys. Rev. B 58, R5948 (1998).

${ }^{8}$ E. Akkermans and K. Mallick, J. Phys. A 32, 7133 (1999).

${ }^{9}$ P.S. Deo, F.M. Peeters, and V.A. Schweigert, Superlattices Microstruct. 25, 1195 (1999).

${ }^{10}$ V.A. Schweigert and F.M. Peeters, Phys. Rev. Lett. 83, 2409 (1999).

${ }^{11}$ M. Zalalutdinov, H. Fujioka, Y. Hashimoto, S. Katsumoto, and Y. Iye, J. Phys. Soc. Jpn. 68, 2872 (1999).

${ }^{12}$ A.K. Geim, S.V. Dubonos, J.J. Palacios, I.V. Grigorieva, M. Henini, and J.J. Schermer, Phys. Rev. Lett. 85, 1528 (2000).

${ }^{13}$ J.J. Palacios, Phys. Rev. Lett. 84, 1796 (2000).

${ }^{14}$ B.J. Baelus, F.M. Peeters, and V.A. Schweigert, Phys. Rev. B 61,
9734 (2000).

${ }^{15}$ F.M. Peeters, V.A. Schweigert, B.J. Baelus, and P.S. Deo, Physica C 332, 255 (2000).

${ }^{16}$ V.A. Schweigert and F.M. Peeters, Physica C 332, 426 (2000).

${ }^{17}$ A.K. Geim, S.V. Dubonos, I.V. Grigorieva, K.S. Novoselov, F.M. Peeters, and V.A. Schweigert, Nature (London) 407, 55 (2000).

${ }^{18}$ B.J. Baelus, F.M. Peeters, and V.A. Schweigert, Phys. Rev. B 63, 144517 (2001).

${ }^{19}$ H.J. Fink and A.G. Presson, Phys. Rev. 151, 219 (1966).

${ }^{20}$ H.J. Fink and A.G. Presson, Phys. Rev. 168, 399 (1968).

${ }^{21}$ G.F. Zharkov, V.G. Zharkov, and A.Yu. Zvetkov, Phys. Rev. B 61, 12293 (2000).

${ }^{22}$ H.J. Fink and W.C.H. Joiner, Phys. Rev. Lett. 23, 120 (1969).

${ }^{23}$ J. O. Indekeu, F. Clarysse, and E. Montevecchi, in $\mathrm{High} \mathrm{T}_{c} \mathrm{Su}$ perconductors and Related Materials, edited by S.-L. Drechsler and T. Mishonov (Kluwer Academic Publishers, Dordrecht, 2001), pp. 487-504.

${ }^{24}$ E. Montevecchi and J.O. Indekeu, Phys. Rev. B 62, 14359 (2000); Europhys. Lett. 51, 661 (2000).

${ }^{25}$ S.V. Yampolskii and F.M. Peeters, Phys. Rev. B 62, 9663 (2000).

${ }^{26}$ P. G. de Gennes, Superconductivity of Metals and Alloys (Addison-Wesley, New York, 1994).

${ }^{27}$ R. Kato, Y. Enomoto, and S. Maekawa, Phys. Rev. B 47, 8016 (1993).

${ }^{28}$ E. Montevecchi, Ph.D. thesis, Katholieke Universiteit Leuven, Leuven, 2000.

${ }^{29}$ A. C. Rose-Innes and E. H. Rhoderick, Introduction to Superconductivity (Pergamon Press, Oxford, 1976). 